\title{
Towards a Common Framework for Mediated Embodiment
}

\author{
Laura Aymerich-Franch ${ }^{*}$ \\ ${ }^{1}$ Ramón y Cajal Senior Research Fellow, Pompeu Fabra University, Barcelona
}

\begin{abstract}
Mediated embodiment is the technologically generated illusion of replacing a person's body with an avatar body. Virtual reality is the most representative technology of mediated embodiment. However, other forms of embodiment are emerging and need to be examined. The inclusion of all mediated embodiment technologies under a common paradigm would more readily facilitate their study. Here, a unified conceptual framework of mediated embodiment is presented, which integrates robot embodiment as part of the phenomenon, and allows the inclusion under the same umbrella of embodiment technologies that might emerge in the future. The minimum conditions necessary to induce the embodiment illusion, as well as the technical principles used to create this illusion, are discussed. Furthermore, it is suggested that mediated embodiment technologies can be regarded as tools that increase human capabilities in four directions: embodiment of a new self; expansion of traveling capabilities; expansion of body capabilities; and the reach of immortality. The principal research conducted in the field of mediated embodiment is explained in connection to these categories. The framework is expected to contribute to creating awareness of the commonalities of mediated embodiment technologies among the different research communities that work with mediated embodiment.
\end{abstract}

Keywords: mediated embodiment, virtual reality, robots, robot embodiment, avatar, body-ownership
Article History

Received 17 December 2019

Revised 29 March 2020

Accepted 29 March 2020

DOI 10.24989/dp.v1i2.1824

\section{Introduction}

Mediated embodiment technologies are capable of generating the illusion that an avatar body temporarily substitutes for a person's body. Experiences of embodiment in avatars are generally associated with virtual reality. However, other technologies can also be used to produce similar illusions. In particular, robot embodiment has recently emerged as another important technology capable of creating this illusion (Alimardani, Nishio, \& Ishiguro, 2013; Aymerich-Franch et al., 2015, 2016, 2017a, 2017b; Aymerich-Franch, Kishore, \& Slater, 2019; Cohen et al., 2012, 2014; Kishore et al., 2014, 2016). While empirical evidence is not yet available, other technologies, such as holograms or drones, could potentially be used to achieve similar effects.

Technologically induced illusions of embodiment can entail important cognitive, attitudinal, and behavioral effects for the embodied user (Aymerich-Franch, Kizilcec, \& Bailenson, 2014; Groom, Bailenson, \& Nass, 2009; Hershfield, et al., 2011; Peck, Seinfeld, Aglioti, \& Slater, 2013; Rosenberg et al., 2013; Won, Bailenson, Lee, \& Lanier, 2015; Yee \& Bailenson, 2007) that need to be examined and understood, especially in the most recent and emerging manifestations of the phenomenon. Thus, a unified conceptual framework of mediated embodiment would help to facilitate the study of this phenomenon as a whole.
The first step is the development of a concept that encompasses all embodiment technologies. This defining concept needs to be broad enough to embrace the illusion of embodiment produced by any type of embodiment technology. At the same time, it needs to be narrow enough to only include experiences of embodiment that are technologically induced.

The concept of mediated embodiment is the most appropriate for this purpose. The term has previously been used in a similar manner to describe the degree to which the user's body is coupled to the interface (Biocca, 2002). Related terminology is also regularly used to describe several processes in virtual reality: users embody avatars, and the feeling of experiencing the avatar's body as one's own during a mediated embodiment experience is called sense of embodiment (Kilteni, Groten, \& Slater, 2012). Notably, virtual embodiment describes the process of mediated embodiment specifically using the technology of virtual reality, that is, employing virtual reality technology to substitute a person's physical body with a virtual one (Spanlang et al., 2014).

Here, mediated embodiment extends the concept of virtual embodiment, and is defined as the technologically-generated illusion of substituting a person's physical body with an avatar, independent of the technology used to produce the illusion (Aymerich-Franch, 2018). Mediated refers to the use of technical mediums (Davis, 2000). Embodiment, as it applies here, indi- 
cates the existence in the world through a body (Csordas, 1999). However, embodiment is a rather complex concept, which has been applied with many different meanings in many different fields and contexts. It remains rather vague as to what exactly it means to be embodied, or how humans and other physical systems are embodied.

Metzinger $(2006,2014)$ proposes a differentiation between first, second, and third-order embodiment. According to this author, first-order embodiment systems are "reactive, adaptive systems, achieving intelligent behavior without explicit computation" (Metzinger, 2014: 272). Second-order embodiment systems "increase their level of causal self-control by explicitly and holistically representing themselves as embodied" (Metzinger, 2014: 274). Finally, third-order embodiment systems are those that "not only explicitly model themselves as an embodied being, but also map some of the representational content generated in this process onto the level of conscious experience" (Metzinger, 2014: 274).

Importantly, in the third-order embodiment, physical systems consciously experience themselves as embodied, while possessing affective or sensorimotor states (Metzinger, 2014). This type of embodiment is found in conscious human beings and also in experimentally induced full-body illusions (Metzinger, 2014). Mediated embodiment experiences with virtual and robotic avatars are a type of experimentally/technologically induced full-body illusion. Hence, they can be considered thirdorder embodiment, according to this classification.

\section{The role of avatars in mediated embodiment}

Avatars are a core element of mediated embodiment. When people are embodied in avatars, they experience ownership and agency over the body of that avatar (Kilteni, Groten, \& Slater, 2012), and self-location within its bodily boundaries (Lenggenhager, Tadi, Metzinger, \& Blanke, 2007; Slater, PerezMarcos, Ehrsson, \& Sanchez-Vives, 2008, 2009; Slater, Spanlang, Sanchez-Vives, \& Blanke, 2010).

Other terms used in place of avatar include "incarnation" or "appearance" (Harper, 2018). In virtual reality, this concept has been largely adopted to describe users' self-representation in the mediated environment (Ahn, Fox, \& Bailenson, 2012). Both its roots and its acceptance in the virtual reality field make the concept perfectly extendable to also describe users' surrogate body in other forms of mediated embodiment. If this wider definition is adopted, then avatars can be classified in two main categories: virtual and physical.

Virtual avatars represent users in virtual reality, videogames, online virtual worlds, social media, and other forms of computer-mediated communication. However, only in immersive virtual reality do users embody the avatar, in the sense that the space generally occupied by the real physical body is replaced by the avatar's virtual body (Spanlang et al., 2014). Generally, ava- tars in virtual reality resemble human bodies, but avatar bodies that represent other entities, such as animals (Ahn, et al., 2016), can also be used for this purpose. Virtual avatars could potentially be used in other emerging technologies, such as in future forms of hologram embodiment.

Physical avatars currently represent users in robotic embodiment. A robot is a physical entity situated in the physical reality. Thus, when users embody a robot, the avatar can be classified as physical. At present, humanoid robots of closely human (Alimardani et al., 2013; Becker-Asano et al., 2012) and non-human appearance (Aymerich-Franch et al., 2015, 2016, 2017a, 2017b; Becker-Asano et al., 2012; Cohen et al., 2012, 2014; Kishore et al., 2014, 2016) are used as physical avatars. However, other physical entities such as drones, other types of robots, or even cyborgs might potentially become physical avatars in future forms of mediated embodiment.

\section{Minimum conditions necessary for experiencing sense of embodiment in mediated embodiment}

Mediated embodiment is related to the process of technologically embodying a user in an avatar, whereas a sense of embodiment in a virtual or physical avatar body is a result of this process.

Kilteni, Groten, and Slater (2012) define sense of embodiment in the specific context of mediated embodiment as "the ensemble of sensations that result from being inside, having, and controlling an avatar body" (p. 374-375).

According to Kilteni, Groten, and Slater (2012), sense of embodiment results from: body-ownership, which is the feeling that a body or a limb belongs to oneself (Gallagher, 2000; Tsakiris, 2010); self-location, which is a determinate volume in space where one feels to be located (Blanke \& Metzinger, 2009); and agency, which refers to the capacity to control one's own actions (Haggard, 2017) or, in the particular case of mediated embodiment, the avatar's actions (Kilteni, Groten, \& Slater, 2012).

The reason why humans are able to experience sense of embodiment in avatar bodies is a complex question. A crucial aspect to understand in relation to this process of re-embodiment is the importance of overcoming the conception of any living being - including humans - as closed, permanent units, at all levels of their existence.

In this regard, it is important to acknowledge the high malleability of human minds and bodies to permanently redefine their boundaries and to incorporate external apparatus as part of their beings (Clark, 2007). This condition of permanent change reaches as far as to the level of self-consciousness. According to the self-model theory, "there is no such thing as a substantial self (as a distinct ontological entity, which could in principle exist by itself), but only a dynamic, ongoing process creating very specific representational and functional properties" (Metzinger, 2007). 
Mediated embodiment setups have been used to provide avatar bodies that extend beyond traditional human appearances, including animals (Ahn et al., 2016), bodies with extra limbs (Schaefer, Heinze \& Rotte, 2009; Won et al., 2015) and tails (Steptoe et al., 2013), or highly robotic-looking avatars (AymerichFranch et al., 2016, 2017a, 2017b; Aymerich-Franch, Kishore, \& Slater, 2019). The sense of embodiment reported by participants in these experiences varies across studies and the limits of embodiment for non-human looking entities remain unclear (Aymerich-Franch \& Ganesh, 2016). The fact that avatar bodies with a closer resemblance to human bodies seem to elicit stronger sense of embodiment suggests that sense of embodiment might be regulated by a top-down perceptual body image that modulates the way in which multisensory information is processed from the bottom-up (Maselli \& Slater, 2013).

The role of body image in leading to sense of embodiment is more specifically discussed by Haans and Ijsselsteijn (2012). They suggest that the three orders of embodiment proposed by Metzinger $(2006,2014)$ can be explained in terms of the morphology of the body - the body schema - which is "a dynamic distributed network of procedures aimed at guiding behavior" (Haans \& Ijsselsteijn, 2012: 213), and the body image, which is a perceptual, cognitive, and/or emotional awareness of the body together with the fact that the body is perceived as owned and as something in itself (Gallagher, 1986). For Haans and Ijsselsteijn (2012), first-order embodiment means having morphology only, second-order embodiment entails having morphology and a body schema, and third-order embodiment implies having morphology, a body schema, and a body image (Haans \& Ijsselsteijn, 2012). In a distinction between functional and phenomenological extensions of the self, Haans and Ijsselsteijn (2012) suggest that when the components of a mediated embodiment system are effectively integrated in the body schema (second-order embodiment), humans can interact with the mediated environment as if the mediating technology was not there. However, feeling embodied in an avatar cannot be explained by incorporation of the avatar into the body schema alone, as it requires consciousness of having a body image, or, in other words, third-order embodiment (Haans \& Ijsselsteijn, 2012).

There are numerous empirical works that contribute to explain how the illusion of embodiment can be artificially induced in experimental contexts. Multisensory correlations are one of the fundamental conditions in this regard. The well-acknowledged rubber-hand illusion experiment (Botvinick \& Cohen, 1998) showed how synchronous touch applied to a hidden real hand and a physical rubber hand visible to the participant led to sense of ownership over the rubber hand.

Similar principles can be applied to create full-body embodiment illusions. For instance, Petkova and Ehrsson (2008) gave participants a first-person perspective (1PP) from a mannequin body and used visuo-tactile synchronization between the mannequin body and the participants' real body to show that ownership illusions also extend to the full-body.
In the specific context of mediated embodiment, there is also empirical evidence that sense of embodiment can be induced in virtual and robotic limbs and bodies when visuo-tactile synchronization is applied between the avatar and the participant's bodies (Aymerich-Franch et al., 2017a; Maselli \& Slater, 2013; Slater, Perez-Marcos, Ehrsson, \& Sanchez-Vives, 2009).

Given the characteristics of mediated embodiment technologies, visuo-motor synchronization is generally used to induce sense of embodiment in avatar bodies (rather than visuo-tactile synchronization). To create visuo-motor synchronization in mediated embodiment systems, the movements of the human user are mapped to the avatar body using real time motion capture (Spanlang et al., 2014).

Other than multi-sensory correlations, a 1PP over the avatar body, which translates also to a spatially coincident location between the real and the avatar body, has also been highlighted as essential for eliciting a body ownership illusion (Maselli \& Slater, 2013; Slater \& Sanchez-Vives, 2014).

While in certain experimental conditions it has been suggested that 1PP alone (Carey, Crucianelli, Preston, \& Fotopoulou, 2019; Maselli \& Slater, 2013; Slater, Spanlang, Sanchez-Vives, \& Blanke, 2010), or multi-sensory correlation alone (Lenggenhager, Tadi, Metzinger, \& Blanke, 2007), might be sufficient to elicit the embodiment illusion, the combination of the two seems crucial to induce sense of embodiment, especially as the resemblance of the avatar body becomes increasingly different to the human body in appearance (Maselli \& Slater, 2013).

In summary, the minimum conditions that need to be satisfied in order to achieve the illusion of mediated embodiment appear to be the following:

- A physical or digital entity that acts as the avatar body

- First-person perspective from the avatar and occluded vision from the real, physical surroundings that result into a spatially coincident location between the avatar body and the physical body of the user (Maselli \& Slater, 2013; Slater \& Sanchez-Vives, 2016; Spanlang et al., 2014)

- Multisensory correlation between the user and the avatar bodies, which is generally translated to visuo-motor synchronization between the user body movements and the avatar movements in mediated embodiment setups (Spanlang et al., 2014)

The implementation of these conditions at the technical level in the principal existing mediated embodiment systems is described below (in section 4: Technical Commonalities of Mediated Embodiment Technologies).

\section{Technical Commonalities of Mediated Embodiment Technologies}

At present, virtual reality and robots are the principal technologies used to create experiences of mediated embodiment. Virtual reality environments are digitally created $3 \mathrm{D}$ spaces in which 
users interact through an avatar. Users' movements are tracked, and their surroundings rendered in accordance with these movements (Fox, Arena, \& Bailenson, 2009). Robots, or more specifically, physical robots, can be broadly defined as autonomous or semi-autonomous machines which are able to sense the environment around them and perform complex tasks within it. Robots that resemble a human body in terms of shape (limbs, head, trunk) are termed "humanoid robots". Teleoperation is the operation of a machine by a person at a distance, in which a human utilizes a master, a manipulator or joystick to give movement commands to the slave (the robot), which performs the task accordingly (Hokayem \& Spong, 2006). The operator (the person controlling the machine or device) has either direct visual contact with the machine or receives visual feedback through a camera mounted on the device. Robot teleoperation could potentially lead to mediated embodiment if the minimum conditions necessary to achieve this illusion are satisfied.

The illusion of mediated embodiment is achieved following a series of technical principles which are common to all embodiment systems. These technicalities are oriented to provide sensory feedback and control of the avatar movements, and satisfy (at least) the minimum necessary conditions for the illusion of mediated embodiment to occur. Concerning sensory feedback, the technical commonalities are the following:

- Visual feedback: As stated earlier, 1PP is a fundamental requirement to induce the illusion of embodiment (GonzálezFranco, et al., 2010; Maselli \& Slater, 2013; Slater et al., 2010). A head-mounted display (HMD) is used to provide visual feedback from the avatar's "eyes" and occlude the participant's view of the real world. In virtual reality, HMDs display the virtual environment whereas in robot embodiment the HMD displays real time video feedback from the robot's eyes. Users are able to see the limbs and part of the body of their avatars if they look down, at the location corresponding to their real limbs. In addition, full-body identification can be achieved by reflecting the avatar's appearance in physi$\mathrm{cal}$ and virtual mirrors or other surfaces (Aymerich-Franch et al., 2016; Aymerich-Franch, Kizilcec, \& Bailenson, 2014; González-Franco, Pérez-Marcos, Spanlang, \& Slater, 2010).

- Feedback from other senses: While feedback from other senses is not a minimum condition to induce the embodiment illusion in the currently existing mediated embodiment technologies, it can be implemented to enhance the experience (Spanlang et al., 2014). Auditory feedback is implemented with the use of headsets or speakers. Haptic feedback, currently utilised less commonly, can be implemented with the aid of different types of haptic devices that facilitate grasping and moving objects, experiencing the feel of a texture, or receiving force feedback (Fox et al., 2009; Stone, 2001). Olfaction and gustation are generally not implemented.

The shared technical principles related to providing control of the avatar's movements (related to the minimum condition of visuomotor synchronization) can be synthetized as follows:
- Head-tracking: In virtual reality embodiment, the movements of the user's head are followed in real-time enabling the system to update the virtual viewpoint based on the data of the tracked head (Spanlang et al., 2014). In robot embodiment, head movements are synchronized to the robot's head movements and users receive video-feedback from cameras mounted on the robot's head in real-time (Aymerich-Franch et al., 2015, 2016, 2017a, 2017b; Kishore et al., 2014, 2016).

- Body-tracking: Users' movements can be tracked and synchronized to the avatar's movements for the control of limb and body gestures and to make the avatar move in the space. In virtual reality, a user's body movement is generally tracked and synchronized to the movement of the avatar body, with spaces rendered according to these movements (Fox et al., 2009; Spanlang et al., 2014). For physical avatars, control of the movement of the robot body can be achieved with a motion capture suit (Aymerich-Franch, Kishore, \& Slater, 2019), a joystick (Aymerich-Franch et al., 2015, 2016), a braincomputer interface (Alimardani et al., 2013; Gergondet et al., 2011), fMRI (Cohen et al., 2012, 2014; Shinkareva et al., 2008), or eye-tracking technologies (Kishore et al., 2014). The ability to control the movement of the avatar body enables the user to discern the boundaries of the embodied body from the surrounding space.

Figure 1 summarizes the minimum conditions necessary for experiencing sense of embodiment and its relation to the technical commonalities of mediated embodiment technologies.

Any technology, in addition to virtual reality and robots (e.g. drones, holograms), that follows these principles and meets the minimum conditions for the mediated embodiment illusion to occur could potentially be used for mediated embodiment. For instance, a mediated embodiment system could be created from a drone provided that the users had a 1PP from the "body" of the drone, their movements were synchronized to those of the drone (e.g. moving the left arm, moves the drone to the left), and the drone presented physical features that were coherent with the body model. Smolyanskiy and Gonzalez-Franco (2017) designed an advanced drone teleoperation system using a fully immersive setup that provided stereoscopic $1 \mathrm{PP}$ through a virtual reality HMD, which could be regarded as a precedent in this regard. Similarly, for a hologram, the illusion of mediated embodiment could potentially take place, provided that a HMD was used which enabled the user controlling the hologram to view its perspective in its surroundings. The users should be able to see the body of their hologram when they look down. Empirical validation, however, is required to prove these assumptions.

It is worth emphasizing that a technology should only be classified as a mediated embodiment technology if it satisfies these minimum criteria. For instance, an avatar in a videogame displayed on a laptop screen would not fall under mediated embodiment, because the avatar and the human body do not have a spatially coincident location. 


\section{Mediated Embodiment}

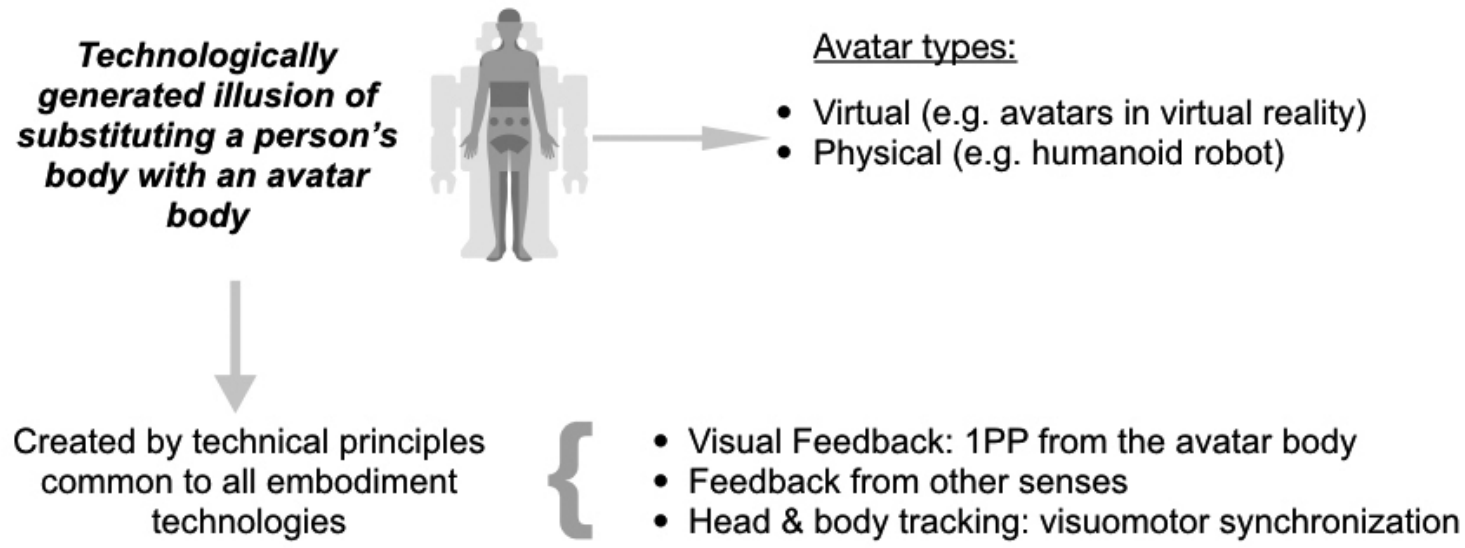

leads to

\section{Sense of embodiment}

Figure 1. The Mediated Embodiment framework

\section{Mediated embodiment as a tool to extend human capabilities}

Since ancient times, human beings have tried to extend their capabilities with the aid of external tools and objects (Berti \& Frassinetti, 2000; Maravita \& Iriki, 2004). Humans integrate these tools as part of their body. There is evidence that when the cerebral representation of body space is extended to include objects or tools used by the person, space previously mapped as far can be remapped as near (Berti \& Frassinetti, 2000). Mediated embodiment technologies can be regarded as an evolution of more primitive means of extending our capacities, such as using a hammer. Hence, it can be seen as a way to extend the human senses (Biocca, 1992), body, and mind (Biocca \& Delaney, 1995).

Based on the principal experimental works conducted to date with mediated embodiment technologies, it is possible to identify four directions towards which mediated embodiment systems enhance these capabilities: embodiment of a new self; expanded travelling capabilities; expanded body capabilities; and immortality. Social scientists need to examine experiences of mediated embodiment in relation to each of these capabilities with the dual purpose of promoting positive uses of mediated embodient and anticipating potential negative outcomes.

\subsection{Embodiment of a new self}

The desire to become someone different using an avatar can be connected to its liberating effects. Mediated embodiment allows users to express their "true mind, the authentic self, unfettered by concerns of self-presentation, or even physical sanction" (Spears \& Lea, 1994, p.430). These experiences give users the opportunity to create new identities and explore what it means to be someone different (Turkle, 1999). Mediated embodiment also gives users the possibility to disassociate the avatar's actions from their real identity, which can reduce feelings of vulnerability regarding self-disclosure, inhibition, or evaluation anxiety, and therefore facilitate social interaction (Suler, 2004; Spears \& Lea, 1994).

When users embody an avatar, their self-representation experiences important transformations. These transformations can be as moderate as changing eye and hair color and as drastic as changing gender (Slater et al., 2010), age (Hershfield et al., 2011), or skin color (Peck et al., 2013). They can even be as extreme as changing "species" to become an animal (Ahn et al., 2016), or a robot (Alimardani et al., 2013; Aymerich-Franch et al. 2015, 2016, 2017a, 2017b; Becker-Asano et al., 2012; Cohen et al., 2012, 2014; Kishore et al., 2014, 2016).

The body plays an important role in shaping how the mind thinks (Clark, 2007; Gallagher, 2005). Thus, being in an avatar's shoes, particularly when it presents substantially different visual characteristics from the real self, may cause important alterations in human behavior. In particular, studies in virtual reality have found that users' behavior is affected by the characteristics of the avatar that they represent (Yee \& Bailenson, 2007). This phenomenon, named Proteus Effect, describes how the appearance of the embodied avatar is able to modify the behavior of the user and it is believed to occur because individuals associ- 
ate certain traits of an avatar with specific behavioral stereotypes and expectations (Yee \& Bailenson, 2007). Therefore, when users believe that others will expect certain behaviors from them because of their avatars' appearance, they engage in those anticipated behaviors (Yee \& Bailenson, 2007).

These findings suggest that mediated embodiment could be successfully used as a method for self-improvement. Use of mediated embodiment environments is considered a safe, controlled, and cost-effective method for treating mental health issues, such as phobias and anxiety-related disorders (Riva, 2005), substance-related disorders, or eating disorders (Freeman et al., 2017). For instance (Aymerich-Franch, Kizilcec, \& Bailenson, 2014) found that modifying the appearance of the avatar so that it looks dissimilar to a user's real appearance can contribute to reducing public speaking anxiety. Falconer et al. (2014, 2016) showed how avatar embodiment can be used to increase selfcompassion and to reduce depression and self-criticism. Also, Osimo, Pizarro, Spanlang, and Slater (2015) and Slater et al. (2019) found that mediated embodiment experiences could be successfully used for self-counselling.

Other findings connected to the benefits of mediated embodiment suggest that these experiences could also be implemented to reduce racial bias (Groom, Bailenson, \& Nass, 2009; Peck et al., 2013), and promote social behavior (Rosenberg, Baughman, \& Bailenson, 2013) or pro-environmental behavior (Ahn et al., 2016; Bailey et al., 2015). On the other hand, mediated embodiment may also engender negative effects, such as inducing aggressive behavior (Calvert \& Tan, 1994), that also need to be examined.

\subsection{Expansion of travelling capabilities}

A particularity of physical avatars is that they can be controlled remotely. Thus, users and physical avatars can be situated in different physical spaces - even in different countries (Cohen et al., 2012, 2014; Kishore et al., 2014). In the future, users embodied in physical avatars could be transported to the most remote places on Earth, into space, or even to other planets. As for virtual avatars, users embodied in these entities have the chance to explore digitally created fantasy worlds that are non-existent in reality. In fact, the expansion of human travelling capabilities is nothing new. Communication technologies such as cinema or television, as well as literature, have performed this function for centuries (Green, Brock, \& Kaufman, 2004). The core and constituting difference of mediated embodiment compared to these precedents is that, in mediated embodiment, users are able, through their avatars, to interact with and in the remote or virtual environments, and their actions have consequences on that environment.

The sense of Presence is a thoroughly researched area in relation to the extended capability of travelling, especially in virtual reality. Presence is defined in virtual reality studies as the sense of being 'there', in the virtual environment (Lombard \& Ditton,
1997). When users feel present in the location of the avatar, they behave and respond emotionally very similarly to how they do in reality (Bailenson et al., 2001; Bailenson et al., 2003; Garau et al., 2005; Sanchez-Vives \& Slater, 2005).

A series of applied uses of mediated embodiment can be identified in connection to the extended capability of transportation. For instance, feeling present in the avatar's environment (and separated from the real environment) can be used as a pain distraction method during an operation or for someone suffering from illness (Malloy \& Milling, 2010; Wiederhold \& Wiederhold, 2007). Likewise, it could also be used for mood management and stress relief, a function largely attributed to communication technologies (Bryant \& Zillmann, 1984; Knobloch, 2003; Zillmann, 1988).

\subsection{Expansion and restitution of body capabilities}

A physical avatar of a robot can possess capabilities far beyond the typical human body. A robot body can be considerably stronger, have the ability to fly or to spend extended periods underwater, resist extremely high or low temperatures and adverse climate conditions as well as toxic, radioactive, and non-oxygen environments, and may have extra and/or longer limbs. The nature of a digital avatar is non-corporeal and therefore free of all constraints linked to corporality such as gravity or perishability.

It is worth noting that even if the resemblance of the avatar to a human body may improve the sense of embodiment (Tsakiris \& Haggard, 2005; Maselli \& Slater, 2013), users are able to experience sense of embodiment in avatars that do not reflect their real appearance (Ahn et al., 2016; Aymerich-Franch, 2012; Steptoe, Steed, \& Slater, 2013; Won et al., 2015). Thus, users can embody avatars that present substantially different body structures from humans and integrate body-parts that do not correspond to their real body structure, such as extra limbs (Won et al., 2015) or tails (Steptoe, Steed, \& Slater, 2013). Research has also found that people embodied in avatars are able to rapidly learn to use a novel body with substantially different body schemas to successfully complete a task (Won et al., 2015).

Avatars, especially of a physical nature, might play an important assistive role in hazardous situations, such as rescue activities, nuclear disasters, or natural catastrophes. In addition, people with mobility impairments and amputees could use avatar bodies for sensorimotor performance, or could control a secondary body to carry out daily routines (e.g. to go shopping or help them to dress). Assistive technologies such as advanced prosthetics or wearable exoskeletons provide precedents in this regard (Cowan et al., 2012).

Mediated embodiment technologies could also be implemented for neurorehabilitation purposes (Perez-Marcos et al., 2012). For instance, techniques such as virtual mirror visual feedback could be useful for motor rehabilitation in people recovering from strokes (Perez-Marcos, 2018). 


\subsection{Immortality}

Physical avatars (principally represented by robots) are made of long-lasting materials such as metal and plastic, and digital avatars are non-corporeal entities which cannot expire. The abiding nature of avatars, in comparison to the perishable flesh-and-bones human body, has led some to envisage avatars as containers of the human mind that can support a perpetual existence (2045Initiative, 2015). This perspective assumes that immortality could be achieved if human consciousness could be transferred to avatar bodies (2045Initiative, 2015). This idea implies re-conceptualizing the notion of the "self" and raises a series of challenging questions: What is the self, exactly? What are the minimal necessary requirements to experience it? And does a mind exists as an independent entity from the body? Similar questions have occupied philosophers and scientists for centuries (Descartes, 1644/1984; James, 1890; Kant, 1781/1999) and still remain unresolved.

In this regard, advanced mediated embodiment systems could be used as methodological tools to study the relationship between body and mind as well as the nature of the self (Ehrsson, 2007; Lenggenhager et al., 2007). Some studies have used mediated embodiment as a method to explore self-consciousness (Aymerich-Franch et al., 2016; Ehrsson, 2007; Guterstam \& Ehrsson, 2012; Lenggenhager et al., 2007; Maselli \& Slater, 2013; Petkova \& Ehrsson, 2008; Slater et al., 2009; van der Hoort, Guterstam, \& Ehrsson, 2011). Other studies have highlighted the usefulness of mediated embodiment experiences to explore (virtual) mortality and near-death experiences (e.g. Barberia et al., 2018).

Given that users experience the properties of the avatars' bodies as if they were their own (de Vignemont, 2011; Kilteni, Groten, \& Slater, 2012), mediated embodiment could also be used as a methodological tool to empirically explore philosophical stances that argue that consciousness and cognition is dependent on the body and the environment (Aymerich-Franch, 2018). One of the most representative theorists in this regard is the phenomenologist philosopher Merleau-Ponty (1945/2002), who defended the body and perception as the primary sources to understand the world. Also, the Embodied Cognition approach sustains that cognition is highly dependent on the characteristics of the physical body and its interactions with the world (Shapiro, 2010; Thelen, Schöner, Scheier, \& Smith, 2001). According to this view, the particular form of embodiment determines the way the environment appears to the agent as well as the way in which the organism can interact with it (Lakoff \& Johnson, 1999; Varela, Thompson, \& Rosch, 1991).

\section{Conclusion}

Mediated embodiment technologies are experiencing a tangible process of democratization. The commercialization of low-cost virtual reality visualization devices exemplifies this process. Systems for robot embodiment might also follow a similar path to reach the market, considering that low cost robots are already available to consumers (e.g. Pepper). The use of drones or holograms for embodiment might potentially evolve as other technologies of mediated embodiment, provided that these technologies apply the minimum conditions necessary to produce the illusion of embodiment.

Experiences of embodiment in these technologies might engender important behavioral, cognitive, and attitudinal effects for users, similar to those identified for avatar embodiment in virtual reality (Aymerich-Franch, Kizilcec, \& Bailenson, 2014; Groom, Bailenson, \& Nass, 2009; Hershfield, et al., 2011; Peck, Seinfeld, Aglioti, \& Slater, 2013; Rosenberg et al., 2013; Won, Bailenson, Lee, \& Lanier, 2015; Yee \& Bailenson, 2007). These consequences are still unknown and need to be carefully examined.

Throughout this article, I have crafted the foundations of a unified framework of mediated embodiment that facilitates the analysis of the emerging mediated embodiment technologies. The framework should also contribute to creating mutual awareness of the commonalities between the work of scholars in different disciplines examining mediated embodiment related topics. Specifically it can highlight links between researchers working in robotic embodiment and robot teleoperation from a Robotics perspective, and those working with virtual reality in the Communication, Media Psychology, or Neuroscience fields. The common framework is expected to facilitate knowledge transfer and collaboration among these and other research communities involved in the research and development of embodiment technologies.

At the core of this framework, I placed the concept of mediated embodiment, which defines the technologically generated illusion of substituting a person's body with an avatar body, independent of the technology used to produce the illusion. I suggested that the minimum conditions necessary to create the mediated embodiment illusion are: a digital or physical avatar to act as a substitute body; 1PP from the avatar and occluded vision from the real surroundings; and visuomotor synchronization of the user' movements to the avatar. Furthermore, I identified a series of technical commonalities across mediated embodiment technologies that are used to create the illusion of embodiment. These commonalities will need to be periodically revised as mediated embodiment technologies evolve.

In the second part of the article, I framed mediated embodiment technologies as an advanced tool to increase human capabilities and identified four directions towards which this technology evolves: the embodiment of a new self; the expansion of traveling capabilities; the expansion of body capabilities; and the reach of immortality. Finally, in addition to understanding the effects of this phenomenon, I emphasized that the scientific community has an important responsibility in promoting positive uses of mediated embodiment technologies (Riva, Baños, Botella, Wiederhold, \& Gaggioli, 2012). I further suggested a series of positive implementations for these technologies within the frame of the four extended human capabilities highlighted.

Finally, mediated embodiment raises important ethical and legal questions that need to be addressed (Aymerich-Franch, Kishore, \& Slater, 2019; Aymerich-Franch \& Fosch-Villaronga, 
2019, 2020; Metzinger, 2013). For instance, shall we consider avatars as part of the self from a legal perspective? (AymerichFranch \& Fosch-Villaronga, 2019). Shall we follow the same moral principles that we use in interacting with a flesh and bone human body, to interact with an avatar? There is evidence to indicate that during mediated embodiment users cognitively and emotionally experience the surroundings of their avatar as if they were their real immediate surroundings (Bailenson et al., 2001, 2003; Garau, Slater, Pertaub, \& Razzaque, 2005; Lee, 2004; Sanchez-Vives \& Slater, 2005), and respond to threats to the avatar as if their real body was actually in danger (González-Franco et al., 2010; Slater et al., 2010). Thus, in a way, it can be assumed that users integrate the avatar's body as part of themselves and that the sense of self expands to this new body. All things considered, the need to maintain interpersonal distance when we interact with an avatar, and the desire to develop legislation to safeguard its integrity, do not seem unjustified (AymerichFranch \& Fosch-Villaronga, 2019). Future research in mediated embodiment will also need to address these matters.

\section{References}

2045 Initiative (2015). Available at <2045.com>. Retrieved March 10th, 2020.

Ahn, S. J. G., Bostick, J., Ogle, E., Nowak, K. L., McGillicuddy, K. T., \& Bailenson, J. N. (2016). Experiencing Nature: Embodying Animals in Immersive Virtual Environments Increases Inclusion of Nature in Self and Involvement with Nature. Journal of Computer-Mediated Communication, 21(6), 399-419.

Ahn, S.J., Fox, J., \& Bailenson, J.N. (2012). Avatars. In Bainbridge, W. S. (Ed.), Leadership in Science and Technology: A Reference Handbook. SAGE Publications.

Alimardani, M., Nishio, S., \& Ishiguro, H. (2013). Humanlike robot hands controlled by brain activity arouse illusion of ownership in operators. Scientific Reports, 3, 2396.

Aymerich-Franch, L. \& Fosch-Villaronga, E. (2020). A self-guiding tool to conduct research with embodiment technologies responsibly. Frontiers in Robotics and AI.

Aymerich-Franch, L., Kishore, S. \& Slater, M. (2019). When Your Robot Avatar Misbehaves You Are Likely to Apologize: An Exploration of Guilt During Robot Embodiment. International Journal of Social Robotics, 1-10.

Aymerich-Franch, L. \& Fosch-Villaronga, E. (2019). What We Learned from Mediated Embodiment Experiments and Why It Should Matter to Policymakers. Presence: Teleoperators and Virtual Environments, 27(1).

Aymerich-Franch, L. (2018). Is mediated embodiment the response to embodied cognition? New Ideas in Psychology, 50.

Aymerich-Franch, L., Petit, D., Ganesh, G., \& Kheddar, A. (2017a). Non-human Looking Robot Arms Induce Illusion of Embodiment. International Journal of Social Robotics, 1-12.

Aymerich-Franch, L., Petit, D., Ganesh, G., \& Kheddar, A. (2017b). Object touch by a humanoid robot avatar induces haptic sensation in the real hand. Journal of Computer-Mediated Communication, 22(4), 215-230.

Aymerich-Franch, L. \& Ganesh, G. (2016). The role of functionality in the body model for self-attribution. Neuroscience Research, 104.
Aymerich-Franch, L., Petit, D., Ganesh, G., \& Kheddar, A. (2016). The second me: Seeing the real body during humanoid robot embodiment produces an illusion of bi-location. Consciousness and Cognition, 46, 99-109.

Aymerich-Franch, L., Petit, D., Ganesh, G., \& Kheddar, A. (2015). Embodiment of a humanoid robot is preserved during partial and delayed control. Proceedings of the IEEE International Workshop on Advanced Robotics and its Social Impacts (ARSO 2015). July, 1-3, Lyon, France.

Aymerich-Franch, L., Kizilcec, R., \& Bailenson, J. (2014). The Relationship between Virtual Self Similarity and Social Anxiety. Frontiers in Human Neuroscience, 8: 944.

Aymerich-Franch, L. (2012). Can we identify with a block? Identification with non-anthropomorphic avatars in virtual reality games. Proceedings of the International Society for Presence Research Annual Conference. October 24-26, Philadelphia, Pennsylvania, USA.

Bailenson, J. N., Blascovich, J., Beall, A. C., \& Loomis, J. M. (2001). Equilibrium Theory Revisited: Mutual Gaze and Personal Space in Virtual Environments. Presence: Teleoperators and Virtual Environments, 10(6), 583-598.

Bailenson, J. N., Blascovich, J., Beall, A. C., \& Loomis, J. M. (2003). Interpersonal distance in immersive virtual environments. Personality and Social Psychology Bulletin, 29(7), 819-833.

Bailey, J.O., Bailenson, J.N., Flora, J., Armel, K.C., Voelker, D., \& Reeves, B. (2015). The impact of vivid and personal messages on reducing energy consumption related to hot water use. Environment and Behavior, 47 (5), 570-592.

Barberia, I., Oliva, R., Bourdin, P., \& Slater, M. (2018). Virtual mortality and near-death experience after a prolonged exposure in a shared virtual reality may lead to positive life-attitude changes. PloS one, 13(11).

Becker-Asano, C., Arras, K., Nebel, B., \& Ishiguro, H. (2012). The effect of anthropomorphism on social tele-embodiment. In IROS 2012 Workshop on Human-Agent Interaction.

Berti, A., \& Frassinetti, F. (2000). When far becomes near: Remapping of space by tool use. Journal of cognitive neuroscience, 12(3), 415-420.

Biocca, F. (1992), Virtual Reality Technology: A Tutorial. Journal of Communication, 42: 23-72.

Biocca, F. (2002). The evolution of interactive media. In M. C. Green, J. J. Strange, \& T. C. Brock (Eds.), Narrative impact. Social and cognitive foundations (pp. 97-130). Taylor \& Francis.

Biocca, F., \& Delaney, B. (1995). Immersive virtual reality technology. In Communication in the age of virtual reality, 57-124.

Blanke, O. (2012). Multisensory brain mechanisms of bodily self-consciousness. Nature Reviews Neuroscience, 13(8), 556.

Blanke, O., \& Metzinger, T. (2009). Full-body illusions and minimal phenomenal selfhood. Trends in cognitive sciences, 13(1), 7-13.

Botvinick, M., \& Cohen, J. (1998). Rubber hands 'feel'touch that eyes see. Nature, 391(6669), 756.

Bryant, J., \& Zillmann, D. (1984). Using television to alleviate boredom and stress: Selective exposure as a function of induced excitational states. Journal of Broadcasting \& Electronic Media, 28(1), 1-20.

Calvert, S. L., \& Tan, S. L. (1994). Impact of virtual reality on young adults' physiological arousal and aggressive thoughts: Interaction versus observation. Journal of Applied Developmental Psychology, 15(1), 125-139.

Carey, M., Crucianelli, L., Preston, C., \& Fotopoulou, A. (2019). The effect of visual capture towards subjective embodiment within the full body illusion. Scientific reports, 9(1), 1-12. 
Clark, A. (2007). Re-inventing ourselves: The plasticity of embodiment, sensing, and mind. Journal of Medicine and Philosophy, 32(3), 263282.

Cohen, O., Druon, S., Lengagne, S., Mendelsohn, A., Malach, R., Kheddar, A., \& Friedman, D. (2012). fMRI Robotic Embodiment: A Pilot Study. In 4th IEEE RAS/EMBS International Conference on Biomedical Robotics and Biomechatronics (BioRob) (pp. 314-319).

Cohen, O., Druon, S., Lengagne, S., Mendelsohn, A., Malach, R., Kheddar, A., \& Friedman, D. (2014). fMRI-Based Robotic Embodiment: Controlling a Humanoid Robot by Thought Using Real-Time fMRI. Presence: Teleoperators and Virtual Environments, 23(3), 229-241.

Cowan, R. E., Fregly, B. J., Boninger, M. L., Chan, L., Rodgers, M. M., \& Reinkensmeyer, D. J. (2012). Recent trends in assistive technology for mobility. Journal of NeuroEngineering and Rehabilitation, 9(1), 20.

Csordas, T. J. (1999). Embodiment and cultural phenomenology. In Perspectives on embodiment: The intersections of nature and culture (pp. 143-162).

Davis, J. E. (2000). Identity and Social Change. Transaction Publishers.

de Vignemont, F. (2011). Embodiment, ownership and disownership. Consciousness and Cognition, 20(1), 82-93.

Descartes, R., (1644/1984). Principles of Philosophy. Kluwer Academic Publishers.

Ehrsson, H. H. (2007). The experimental induction of out-of-body experiences. Science, 317(5841), 1048.

Falconer, C. J., et al. (2016). Embodying self-compassion within virtual reality and its effects on patients with depression. British Journal of Psychiatry Open. http://doi.org/10.1192/bjpo.bp.115.002147

Falconer, C. J., Slater, M., Rovira, A., King, J. A., Gilbert, P., Antley, A., \& Brewin, C. R. (2014). Embodying compassion: A virtual reality paradigm for overcoming excessive self-criticism. PLoS ONE. https://doi.org/10.1371/journal.pone.0111933

Fox, J., Arena, D., \& Bailenson, J. N. (2009). Virtual Reality: A Survival Guide for the Social Scientist. Journal of Media Psychology, 21(3), 95-113.

Gallagher, S. (2005). How the body shapes the mind. Oxford: Clarendon Press.

Gallagher, S. (2000). Philosophical conceptions of the self: implications for cognitive science. Trends in cognitive sciences, 4(1), 14-21.

Garau, M., Slater, M., Pertaub, D. P., \& Razzaque, S. (2005). The responses of people to virtual humans in an immersive virtual environment. Presence: Teleoperators and Virtual Environments, 14(1), 104-116.

Gergondet, P., Druon, S., Kheddar, A., Hintermüller, C., Guger, C., \& Slater, M. (2011, December). Using brain-computer interface to steer a humanoid robot. In Robotics and Biomimetics (ROBIO), 2011 IEEE International Conference on (pp. 192-197). IEEE.

González-Franco, M., Pérez-Marcos, D., Spanlang, B., \& Slater, M. (2010, March). The contribution of real-time mirror reflections of motor actions on virtual body ownership in an immersive virtual environment. In Virtual Reality Conference (VR), 2010 IEEE (pp. 111-114). IEEE.

Green, M. C., Brock, T. C., \& Kaufman, G. F. (2004). Understanding Media Enjoyment: The Role of Transportation Into Narrative Worlds. Communication Theory, 14, 311-327.

Groom, V., Bailenson, J. N., \& Nass, C. (2009). The influence of racial embodiment on racial bias in immersive virtual environments. Social Influence, 4(3), 231-248.

Guterstam, A., \& Ehrsson, H. H. (2012). Disowning one's seen real body during an out-of-body illusion. Consciousness and Cognition, 21(2), $1037-42$.

Haggard, P. (2017). Sense of agency in the human brain. Nature Reviews Neuroscience. https://doi.org/10.1038/nrn.2017.14

Haans, A., \& Ijsselsteijn, W. A. (2012). Embodiment and telepresence: Toward a comprehensive theoretical framework 2 . Interacting with Computers, 24(4), 211-218.

Harper. D. (2018). Online Etymology Dictionary. Retrieved June 26, 2018, from http://www.etymonline.com/

Hershfield, H. E., Goldstein, D. G., Sharpe, W. F., Fox, J., Yeykelis, L., Carstensen, L. L., \& Bailenson, J. N. (2011). Increasing saving behavior through age-progressed renderings of the future self. Journal of Marketing Research, 48(SPL), S23-S37.

Hokayem, P. F., \& Spong, M. W. (2006). Bilateral teleoperation: An historical survey. Automatica, 42(12), 2035-2057.

James, W. (1890). The principles of psychology (Vols. 1 \& 2). New York Holt, 118, 688.

Kant, I. (1781/1999). Critique of Pure Reason (Vol. 2). Guyer, P. \& Wood, A.W. (Eds.). Cambridge University Press.

Kilteni, K., Groten, R., \& Slater, M. (2012). The Sense of Embodiment in Virtual Reality. Presence: Teleoperators and Virtual Environments, 21(4), 373-387.

Kishore, S., González-Franco, M., Hintemüller, C., Kapeller, C., Guger, C., Slater, M., \& Blom, K. J. (2014). Comparison of SSVEP BCI and Eye Tracking for Controlling a Humanoid Robot in a Social Environment. Presence: Teleoperators and Virtual Environments, 23(3), 242-252.

Kishore, S., Navarro, X., Dominguez, E., De La Peña, N., \& Slater, M. (2016). Beaming into the News: A System for and Case Study of TeleImmersive Journalism, IEEE Computer Graphics and Applications, 99.

Knobloch, S. (2003). Mood adjustment via mass communication. Journal of communication, 53(2), 233-250.

Lakoff, G., \& Johnson, M. (1999). Philosophy In the Flesh: The Embodied Mind And Its Challenge To Western Thought. New York, NY: Basic Books.

Lee, K. M. (2004). Presence, explicated. Communication Theory, 14(1), 27-50.

Lenggenhager, B., Tadi, T., Metzinger, T., \& Blanke, O. (2007). Video ergo sum: manipulating bodily self-consciousness. Science (New York, N.Y.), 317(5841), 1096-9.

Lombard, M., \& Ditton, T. (1997). At the heart of it all: The concept of presence. Journal of Computer-Mediated Communication, 3(2), 0-0.

Longo, M. R., Schüür, F., Kammers, M. P., Tsakiris, M., \& Haggard, P. (2008). What is embodiment? A psychometric approach. Cognition, 107(3), 978-998.

Malloy, K. M., \& Milling, L. S. (2010). The effectiveness of virtual reality distraction for pain reduction: a systematic review. Clinical psychology review, 30(8), 1011-1018.

Maravita, A., 2\& Iriki, A. (2004). Tools for the body (schema). Trends in cognitive sciences, 8(2), 79-86.

Maselli, A., \& Slater, M. (2013). The building blocks of the full body ownership illusion. Frontiers in Human Neuroscience, 7(March), 83.

Merleau-Ponty, M. (1965). The Phenomenology of Perception. Routledge \& Kegan Paul. London: Routledge \& Kegan Paul.

Metzinger, T. (2006). Reply to Gallagher: Different conceptions of embodiment. PSYCHE: An Interdisciplinary Journal of Research On Consciousness_ 12 . 
Metzinger, T. (2007). Self models. Scholarpedia, 2(10), 4174.

Metzinger, T. (2013). Two principles for robot ethics. In E. Hilgendorf \& J.-P. Günther (Hrsg.), Robotik und Gesetzgebung. Baden-Baden: Nomos. S. 263-302.

Metzinger, T. (2014). First-order embodiment, second-order embodiment, third-order embodiment. In L. Shapiro (Ed.), Routledge handbooks in philosophy. The Routledge handbook of embodied cognition (p. 272-286). Routledge/Taylor \& Francis Group.

Osimo, S. A., Pizarro, R., Spanlang, B., \& Slater, M. (2015). Conversations between self and self as Sigmund Freud - A virtual body ownership paradigm for self counselling. Scientific Reports. http://doi. org/10.1038/srep13899

Peck, T. C., Seinfeld, S., Aglioti, S. M., \& Slater, M. (2013). Putting yourself in the skin of a black avatar reduces implicit racial bias. Consciousness and cognition, 22(3), 779-787.

Petkova, V. I., \& Ehrsson, H. H. (2008). If I were you: perceptual illusion of body swapping. PloS One, 3(12), e3832.

Perez-Marcos, D. (2018). Virtual reality experiences, embodiment, videogames and their dimensions in neurorehabilitation. Journal of neuroengineering and rehabilitation, 15(1), 1-8.

Perez-Marcos, D., et al. (2012). A fully immersive set-up for remote interaction and neurorehabilitation based on virtual body ownership. Frontiers in neurology, 3, 110.

Riva, G. (2005). Virtual reality in psychotherapy: review. Cyberpsychology \& Behavior, 8(3), 220-230; discussion 231-240.

Riva, G., Baños, R. M., Botella, C., Wiederhold, B. K., \& Gaggioli, A. (2012). Positive Technology: Using Interactive Technologies to Promote Positive Functioning. Cyberpsychology, Behavior, and Social Networking, 15(2), 69-77.

Rosenberg, R. S., Baughman, S. L., \& Bailenson, J. N. (2013). Virtual Superheroes: Using Superpowers in Virtual Reality to Encourage Prosocial Behavior. PLoS ONE, 8(1), 1-9.

Sanchez-Vives, M. V., \& Slater, M. (2005). From presence to consciousness through virtual reality. Nature Reviews Neuroscience, 6(4), 332-339.

Schaefer, M., Heinze, H. J., \& Rotte, M. (2009). My third arm: Shifts in topography of the somatosensory homunculus predict feeling of an artificial supernumerary arm. Human Brain Mapping, 30(5), 1413-1420.

Shapiro, L. (2010). Embodied Cognition. Routledge.

Shinkareva, S. V, Mason, R. a, Malave, V. L., Wang, W., Mitchell, T. M., \& Just, M. A. (2008). Using FMRI brain activation to identify cognitive states associated with perception of tools and dwellings. PloS One, 3(1), e1394.

Slater, M., Perez-Marcos, D., Ehrsson, H. H., \& Sanchez-Vives, M. V. (2008). Towards a digital body: the virtual arm illusion. Frontiers in Human Neuroscience, 2(August), 6.

Slater, M., Perez-Marcos, D., Ehrsson, H. H., \& Sanchez-Vives, M. V. (2009). Inducing illusory ownership of a virtual body. Frontiers in Neuroscience, 3(2), 214-20.

Slater, M., Spanlang, B., Sanchez-Vives, M. V, \& Blanke, O. (2010). First person experience of body transfer in virtual reality. PloS One, 5(5), e10564.

Slater, M., \& Sanchez-Vives, M. V. (2016). Enhancing Our Lives with Immersive Virtual Reality. Frontiers in Robotics and AI, 3, 74.

Slater, M., Neyret, S., Johnston, T., Iruretagoyena, G., de la Campa Crespo, M. Á., Alabèrnia-Segura, M., ... \& Feixas, G. (2019). An experimental study of a virtual reality counselling paradigm using embodied self-dialogue. Scientific reports, 9(1), 1-13.
Smolyanskiy, N., \& Gonzalez-Franco, M. (2017). Stereoscopic first person view system for drone navigation. Frontiers in Robotics and AI, 4, 11.

Spanlang, B., Normand, J. M., Borland, D., Kilteni, K., Giannopoulos, E., Pomés, A., ... \& Slater, M. (2014). How to build an embodiment lab: achieving body representation illusions in virtual reality. Front. Robot. AI, 1(9).

Spears, R., \& Lea, M. (1994). Panacea or panopticon? The hidden power in computer-mediated communication. Communication Research, 21 (4), 427-459.

Steptoe, W., Steed, A., \& Slater, M. (2013). Human tails: ownership and control of extended humanoid avatars. IEEE Transactions on Visualization and Computer Graphics, 19(4), 583-90.

Stone, R. J. (2001). Haptic feedback: A brief history from telepresence to virtual reality. Haptic Human-Computer Interaction, 1-16.

Suler, J. (2004). The online disinhibition effect. CyberPsychology and Behavior, 7, 321-326.

Thelen, E., Schöner, G., Scheier, C., \& Smith, L. B. (2001). The dynamics of embodiment: a field theory of infant perseverative reaching. The Behavioral and Brain Sciences, 24(1), 1-34; discussion 34-86.

Tsakiris, M. (2010). My body in the brain: a neurocognitive model of body-ownership. Neuropsychologia, 48(3), 703-712.

Tsakiris, M., \& Haggard, P. (2005). The rubber hand illusion revisited: visuotactile integration and self-attribution. Journal of Experimental Psychology, 31(1), 80-91.

Turkle, S. (1999). Cyberspace and Identity. Contemporary Sociology, 28 (6), 643-648.

van der Hoort, B., Guterstam, A., \& Ehrsson, H. H. (2011). Being Barbie: the size of one's own body determines the perceived size of the world. PloS One, 6(5), e20195.

Varela, F. J., Thompson, E., \& Rosch, E. (1991). The Embodied Mind: Cognitive Science and Human Experience. Cambridge, MA: The MIT Press.

Wiederhold, M. D., \& Wiederhold, B. K. (2007). Virtual reality and interactive simulation for pain distraction. Pain Medicine, 8(SUPPL.3).

Won, A.S., Bailenson, J.N., Lee, J.D., Lanier, J. (2015). Homuncular Flexibility in Virtual Reality. Journal of Computer-Mediated Communication, 20, 241-259.

Yee, N. \& Bailenson, J.N. (2007). The Proteus Effect: Self transformations in virtual reality. Human Communication Research, 33 (3), 271-290.

Zillmann, D. (1988). Mood management: Using entertainment to full advantage. Communication, social cognition, and affect, 31, 147-171.

\section{${ }^{*}$ Corresponding Author}

\section{Laura Aymerich-Franch}

Ramón y Cajal Senior Research Fellow, Pompeu Fabra University C/Roc Boronat, 138, 08018 Barcelona

ORCID ID: 0000-0001-7627-5770, laura.aymerich@gmail.com

\section{Conflict of Interest}

LAF declares no conflict of interest.

\section{Funding}

LAF is supported by the Ramón y Cajal Fellowship Program (ref. RYC2016-19770), funded by Agencia Estatal de Investigación, Ministerio de Ciencia, Innovación y Universidades, and the European Social Fund. 